\title{
Anisotropic Strain on GaN Microdisks Grown by Plasma-Assisted Molecular Beam Epitaxy
}

\author{
Hong-Yi Yang ${ }^{1}$, Ikai Lo ${ }^{1, * \mathbb{D}}$, Cheng-Da Tsai ${ }^{1}$, Ying-Chieh Wang ${ }^{1}$, Huei-Jyun Shih ${ }^{1}$, \\ Hui-Chun Huang ${ }^{1}$, Mitch M. C. Chou ${ }^{1}$, Louie Huang ${ }^{2}$, Terence Wang ${ }^{2}$ and Ching T. C. Kuo ${ }^{2}$ \\ 1 Department of Physics, Department of Materials and Optoelectronic Science, Center for Nanoscience and \\ Nanotechnology, National Sun Yat-Sen University, Kaohsiung 80424, Taiwan; \\ d042030008@student.nsysu.edu.tw (H.-Y.Y.); d002030010@student.nsysu.edu.tw (C.-D.T.); \\ d982030006@student.nsysu.edu.tw (Y.-C.W.); d052030002@student.nsysu.edu.tw (H.-J.S.); \\ m9036601@student.nsysu.edu.tw (H.-C.H.); mitch@faculty.nsysu.edu.tw (M.M.C.C.) \\ 2 Advanced Semiconductor Engineering, Inc., Kaohsiung 811, Taiwan; louie_huang@aseglobal.com (L.H.); \\ Terence_Wang@aseglobal.com (T.W.); ChingTC_Kuo@aseglobal.com (C.T.C.K.) \\ * Correspondence: ikailo@mail.nsysu.edu.tw
}

Received: 18 September 2020; Accepted: 2 October 2020; Published: 4 October 2020

\begin{abstract}
Lattice relaxation on wurtzite GaN microdisks grown by plasma-assisted molecular beam epitaxy was systematically studied. The lattice constants of GaN microdisks were evaluated from high-resolution transmission electron microscopy, and the anisotropic strain was then analyzed by observing the microscopic atomic layers. We found that the vertical lattice strain along the c-axis followed a linear relationship, while the lateral lattice strain along the a-axis exhibited a quadratic deviation. The lattice mismatch is about $0.94 \%$ at the interface between the GaN microdisks and the $\gamma-\mathrm{LiAlO}_{2}$ substrate, which induces the anisotropic strain during epi-growth.
\end{abstract}

Keywords: plasma-assisted molecular beam epitaxy; GaN-microdisks; anisotropic strain

\section{Introduction}

Group-III nitride compounds have been extensively investigated in recent years due to their promising applications in spintronic devices [1,2] and optoelectronic lighting sources [3,4]. Because the lattice constants are quite different among these binary compounds, i.e., AlN $(a=3.11 \AA, c=4.98 \AA)$, GaN $(a=3.160 \AA, c=5.125 \AA)$ and InN $(a=3.5446 \AA, c=5.7034 \AA)$ [5]. The hetero-structured quantum wells $(\mathrm{QW})$ made of their ternary alloys, such as $\mathrm{Al}_{x} \mathrm{Ga}_{1-\mathrm{x}} \mathrm{N} / \mathrm{GaN}$ or $\mathrm{GaN} / \mathrm{In}_{\mathrm{x}} \mathrm{Ga}_{1-\mathrm{x}} \mathrm{N}$ QWs, always produce a strain at the hetero-interface due to the lattice mismatch. The lattice-mismatched strain can induce a piezoelectric field at the hetero-interface of the $\mathrm{Al}_{x} \mathrm{Ga}_{1-\mathrm{x}} \mathrm{N} / \mathrm{GaN} \mathrm{QW}$ to enhance the spin-orbital interaction for spintronic qubit applications [1,2]. However, the lattice-mismatched strain will also degrade the quality of $\mathrm{GaN} / \mathrm{In}_{x} \mathrm{Ga}_{1-x} \mathrm{~N}$ QWs, and hence degrade its optical performance, limiting the application of high-indium alloyed $\mathrm{GaN} / \mathrm{In}_{x} \mathrm{Ga}_{1-x} \mathrm{~N}$ QWs [6-8]. This obstacle limits the application of $\mathrm{GaN} / \mathrm{In}_{x} \mathrm{Ga}_{1-\mathrm{x}} \mathrm{N}$ QW for a full-spectrum LED display. A possible workaround has been proposed by using blue light from the GaN/ $/ n_{x} G_{1-x} N(x \sim 0.13)$ QW light-emitting diode (LED) and mixing with phosphor to produce a white lighting source [3,4]. Currently, the trend of LED displays the reduction in red-green-blue (RGB) LED pixel size from mini-meters down to micro-meters. There are two approaches to manufacturing the RGB micro-LED display: one is a direct photo-lithographic mini-meter/micro-meter process on $\mathrm{GaN} / \mathrm{In}_{\mathrm{x}} \mathrm{Ga}_{1-\mathrm{x}} \mathrm{N}$ epi-film, while the other is a direct epi-growth of $\mathrm{GaN} / \mathrm{In}_{x} \mathrm{Ga}_{1-x} \mathrm{~N}$ microdisk, previously developed by our group [9-11]. In the photo-lithographic processing approach, one limitation is that the smaller the mini/micro-LED lithographed from epi-film, the worse the edge damage on the mini/micro-LED that occurs. Therefore, Lo et al. developed a 
3-dimensional (3D), low-temperature, self-assembling, epi-growth of hexagonal c-plane GaN microdisks on $\gamma-\mathrm{LiAlO}_{2}$ (LAO) substrates by plasma-assisted molecular beam epitaxy (PAMBE) [9]. The 3D GaN microdisk can be treated as a nearly free-standing $\mathrm{GaN}$ substrate for the further self-assembling growth of $\operatorname{In}_{x} \mathrm{Ga}_{1-x} \mathrm{~N} / \mathrm{GaN}$ QW $[10,11]$ and RGB LED [12]. Because the size of a 3D GaN microdisk can be controlled to $1 \sim 4 \mu \mathrm{m}$ with high-quality $\operatorname{In}_{\mathrm{x}} \mathrm{Ga}_{1-\mathrm{x}} \mathrm{N} / \mathrm{GaN} \mathrm{QWs}$, a full-spectrum RGB micro-LED display can be achieved simply by engineering the indium-content of the $\operatorname{In}_{x} \mathrm{Ga}_{1-x} \mathrm{~N} / \mathrm{GaN}$ QW. By doing so, the existence of lattice-mismatched strain allows for easier manufacture of high-indium content $\mathrm{In}_{\mathrm{x}} \mathrm{Ga}_{1-\mathrm{x}} \mathrm{N} / \mathrm{GaN}$ micro-LEDs but requires theoretical re-calculation of the $\operatorname{In}_{\mathrm{x}} \mathrm{Ga}_{1-\mathrm{x}} \mathrm{N} / \mathrm{GaN} \mathrm{QW}$ band structure. In this paper, we study the lattice relaxation of the microstructure on wurtzite GaN microdisks grown by PAMBE. The strain effect on the GaN microdisk is evaluated from the lattice relaxation by high-resolution transmission electron microscopy (TEM), and the microscopic atomic layers are analyzed.

\section{Materials and Methods}

Three $c$-plane $(000 \overline{1}) \mathrm{GaN}$ microdisk samples were grown on $1 \times 1 \mathrm{~cm}^{2} \gamma$-LAO substrates by PAMBE (Veeco Applied-GEN 930 system (White Bear Lake, Minnesota)) with standard effusion cells for Ga-evaporation and an rf-plasma cell with $450 \mathrm{~W}$ for the N2-plasma source. The LAO substrates were cleaned with acetone (5 $\mathrm{min})$, isopropanol (5 min), phosphoric acid (1:30) (5 min), de-ionized water $(5 \mathrm{sec})$, and then were sequentially dried by nitrogen gas. After the cleaning, the LAO substrates were mounted on a holder and out-gassed in an MBE chamber at $700{ }^{\circ} \mathrm{C}$ for 10 minutes. Thereafter, the substrate temperature was decreased to the growth temperature. The detail of the epi-growth can be accessed in our previous papers $[9,10]$. The Ga wetting layer was deposited on the LAO substrate for 5 minutes at, and then the GaN microdisk samples were grown at three temperatures: 620, 630 and $640{ }^{\circ} \mathrm{C}$ (denoted as samples A, B and C, respectively) with the same (N:Ga) flux ratio of $\left(9.0 \times 10^{-6}\right.$ torr:6.5 $\times 10^{-8}$ torr) for 70 minutes. Because the surface diffusion of $\mathrm{GaN}$ epi-growth is a function of growth temperature, the effect of different temperature is then under investigation as well. Three GaN microdisks with a diameter of $\sim 1.5 \mu \mathrm{m}$ were selected from samples $\mathrm{A}, \mathrm{B}$, and $\mathrm{C}$ for the study of microscopic lattice relaxation. The scanning electron microscope (SEM) images from the top view and a tilted angle of the samples focused on the hexagonal GaN microdisks are shown in Figure 1.

The transmission electron microscope (TEM) specimens were prepared by a dual-beam focus ion beam (FIB) with a cleavage face along the [1100] direction, as shown by the dashed lines in Figure 1a,d,g. Based on the ball-stick model of GaN microdisk, as shown in the inset of Figure 1c [9], the lateral width in the [1100] direction per primitive cell is equal to the spacing between $\{1 \overline{1} 00\}$ planes, $d_{M}=\sqrt{3} a / 2$, and this spacing between $\{000 \overline{1}\}$ planes is just equal to lattice constant $c, \boldsymbol{d}_{\boldsymbol{c}}=\boldsymbol{c}$, where $\boldsymbol{a}$ and $\boldsymbol{c}$ are the lattice constants of wurtzite GaN. The edge of oblique surface (1101) is then tilted off the $c$-axis by the angle $=\tan ^{-1}\left(d_{M} / d_{c}\right)=\tan ^{-1}(\sqrt{3} a / 2 c)=28.1^{\circ}$ (we used $a=0.3160 \mathrm{~nm}$, and $c=0.5125 \mathrm{~nm}$ for the ideal bulk GaN wurtzite) [5]. The angle of $28^{\circ}$ can be easily checked on the TEM images of the three samples shown in Figure 1c,f,i. However, the 3D divergent self-assembling growth of the GaN microdisk will generate unbalanced stress between $\{000 \overline{1}\}$ planes during the epi-growth by PAMBE, finally leaving dangling bonds on the oblique surfaces of (1101) and the top (0001) surface. On the epi-growth of the GaN microdisk, the unbalanced stress, therefore, yielded a lattice relaxation during the growth of the layer-by-layer self-assembly. In order to investigate the lattice relaxation, we performed the high-resolution field-emission TEM measurement (Tecnai F20G2 MAT S-TWIN) at the locations marked with vertical lines along the $c$-axis and the lateral line along the [1100] direction, shown in Figure 1c,f,i. The lattice constants were then calculated from the microscopic TEM images. 

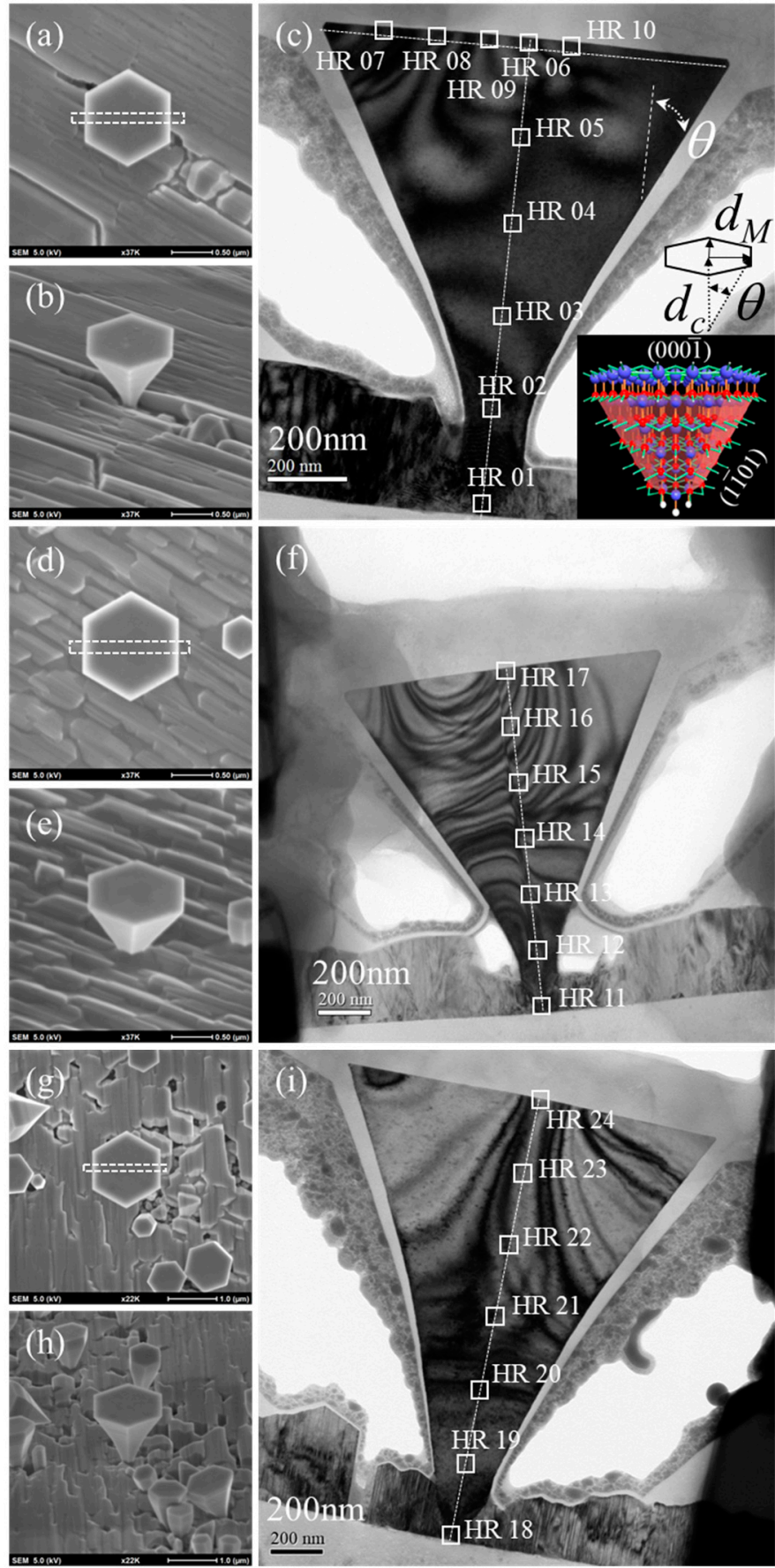

Figure 1. (a,d,g) SEM top-view images of samples A, B, and C, respectively, with the cleavage plane along $[1 \overline{1} 00]$ direction (scale bars are $0.5 \mu \mathrm{m}$ for A and B, $1 \mu \mathrm{m}$ for C). (b,e,h) SEM images of $(\mathbf{a}, \mathbf{d}, \mathbf{g})$ at a tilted angle. (c,f,i) TEM images of samples A, B, and C (scale bars are $0.2 \mu \mathrm{m}$ ). Inset of (c) shows a ball-stick model of GaN microdisk, and the $d$-spacing of primitive cell.

\section{Results and Discussion}

The high-resolution TEM measurement was performed for sample A on locations HR01-HR06 along the $c$-axis and lateral locations HR07-HR10 along the [1100] direction, shown in Figure 1c. The high-resolution TEM image of spot HR01 is shown in Figure 2a1. The d-spacing can be calculated from atomic layers by scanning the microscopic structure of this high-resolution TEM image using the internal software "DigitalMicrograph" provided by Tecnai, Inc. We scanned ten successive lattice lines along the [1100] direction for $\mathrm{dM}$-spacing and c-axis for dc-spacing, as marked by yellow lines in 
Figure 2a1, and took an average value for the location (e.g., at height $\mathrm{z}$ ). The examples of scanned lattice lines are shown in Figure 2a2 (horizontal) and Figure 2a3 (vertical) for $\boldsymbol{d}_{\boldsymbol{M}}$-spacing and $\boldsymbol{d}_{\boldsymbol{c}}$-spacing, respectively. We then obtained the averages $d_{M}=0.2742 \mathrm{~nm}$ and $d c=0.52408 \mathrm{~nm}$ at HR01, yielding the angle $\theta=\tan ^{-1}\left(d_{M} / d_{c}\right)=27.62^{\circ}$ near the initial nucleation of GaN atop the LAO substrate. Similar analyses were repeated for HR02-HR06, some of which are shown in Figure 2b for HR02, Figure 2c for HR04, and Figure 2d for HR06. For instance, we obtained the average $d_{M}=0.2748 \mathrm{~nm}$ and $d_{c}=0.5237 \mathrm{~nm}$ for HR02, giving $\theta=27.69^{\circ}$. For HR04, we obtained average $d_{M}=0.2764 \mathrm{~nm}$ and $\boldsymbol{d}_{\boldsymbol{c}}=0.5241 \mathrm{~nm}$, yielding $\theta=27.81^{\circ}$. For HR06, we obtained average $\boldsymbol{d}_{\boldsymbol{M}}=0.2771 \mathrm{~nm}$ and $\boldsymbol{d}_{\boldsymbol{c}}=0.5241 \mathrm{~nm}$, yielding $\theta=27.87^{\circ}$. The lattice strain is relaxed along the $c$-axis with the angle spreading from 27.62 to $27.81^{\circ}$ during the epi-growth from the substrate up to the top surface.
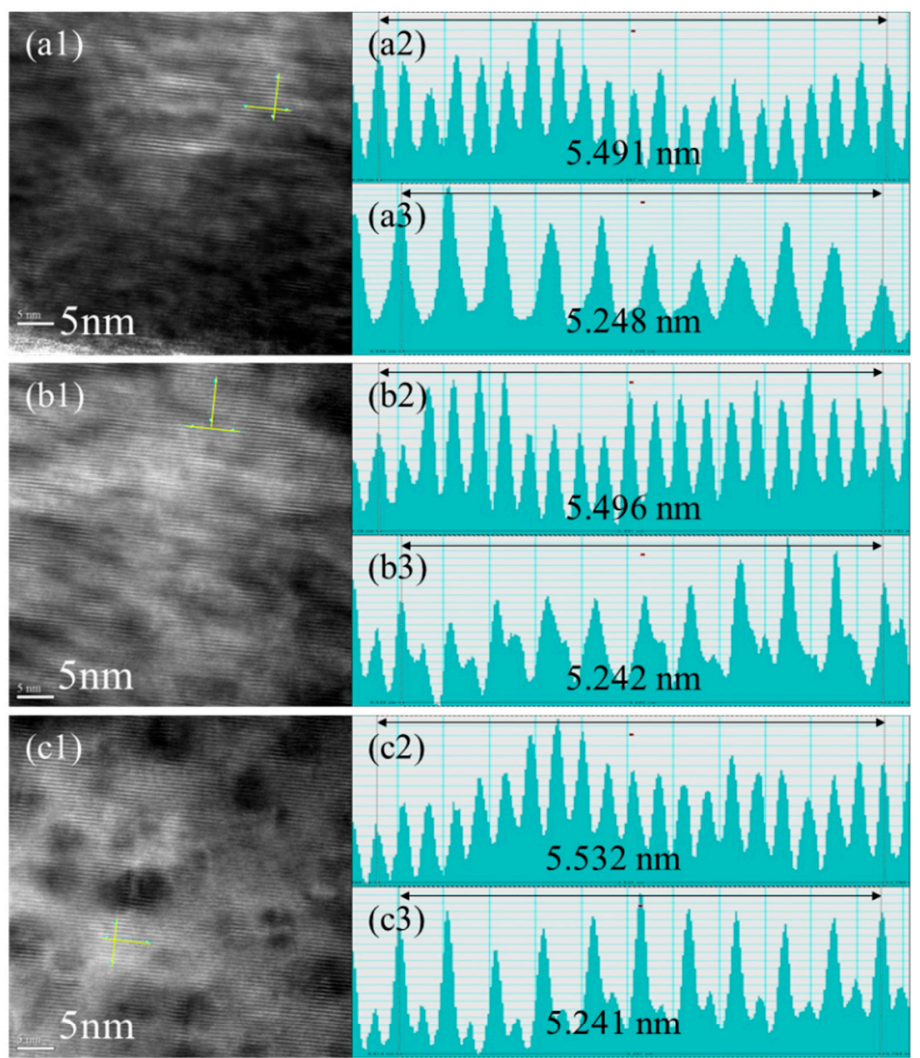

(c2)

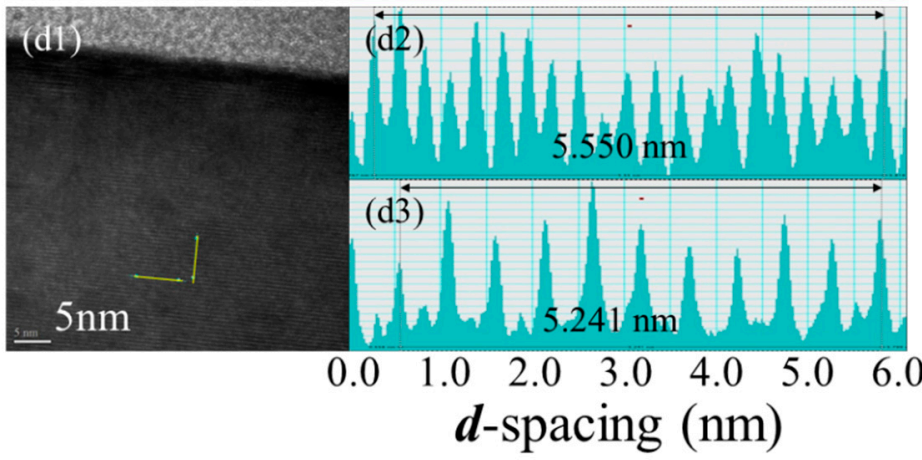

Figure 2. (a1,b1,c1,d1) High-resolution TEM images at HR01, HR02, HR04, and HR06, with scale bar of $5 \mathrm{~nm}$. (a2,a3) The $d_{M^{-}}$and $d_{c^{-}}$-spacing measurements for (a1). (b2,(b3) The $d_{M^{-}}$and $d_{c^{-}}$-spacing measurements for (b1). (c2,c3) The $\boldsymbol{d}_{\boldsymbol{M}^{-}}$and $\boldsymbol{d}_{\mathcal{c}^{-}}$-spacing measurements for (c1). (d2,d3) The $\boldsymbol{d}_{\boldsymbol{M}^{-}}$and $d_{c}$-spacing measurements for (d1).

The lateral relaxation at the top surface was also evaluated for HR07-HR10, as shown in Figure $3 a-d$, respectively. 

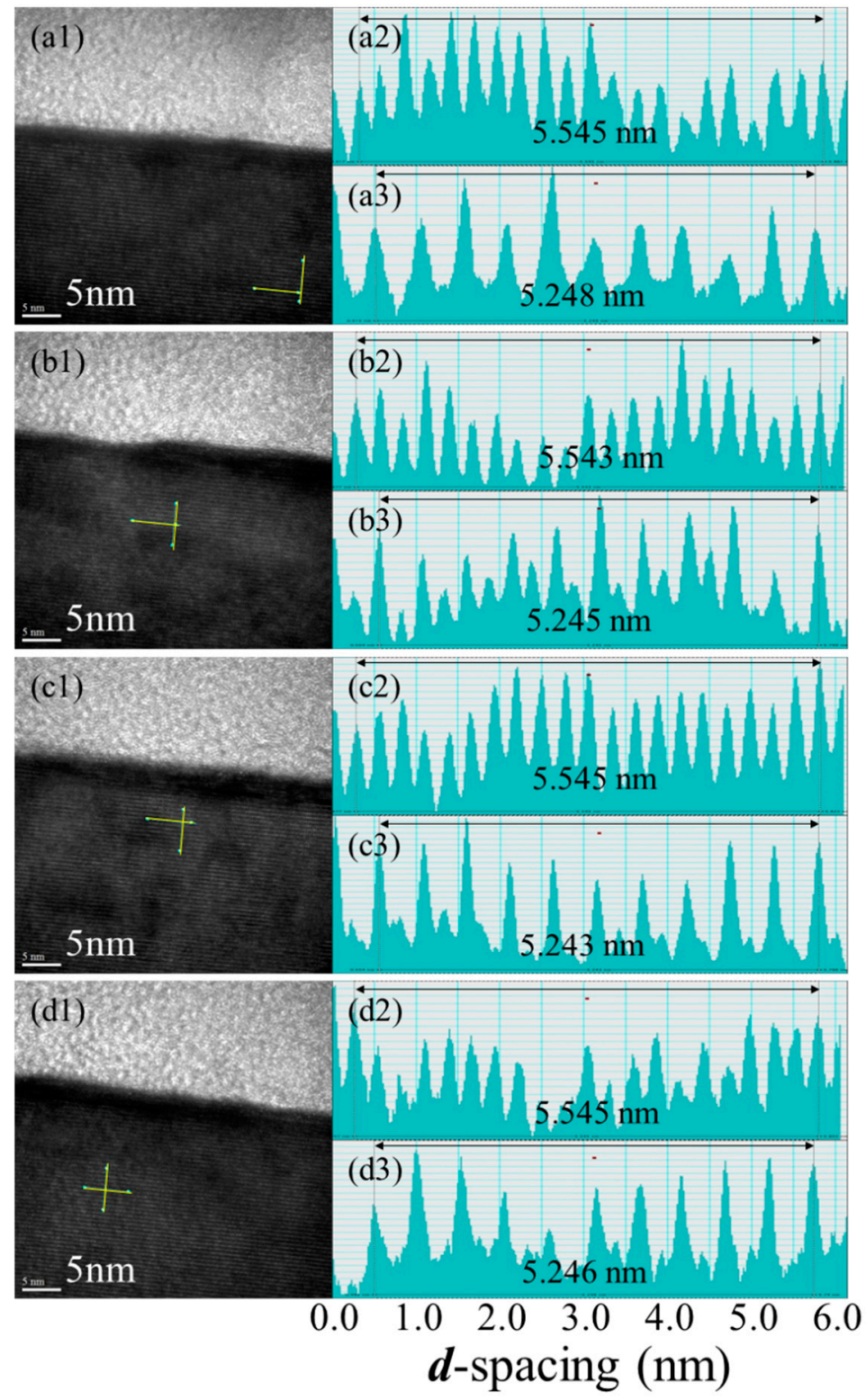

Figure 3. (a1,b1,c1,d1) High-resolution TEM images at HR07, HR08, HR09, and HR10, with scale bar of $5 \mathrm{~nm}$. (a2,a3) The $\boldsymbol{d}_{M^{-}}$and $\boldsymbol{d}_{\mathcal{c}^{-}}$-spacing measurements for $(\mathbf{a} 1) .(\mathbf{b} 2, \mathrm{~b} 3)$ The $\boldsymbol{d}_{M^{-}}$and $\boldsymbol{d}_{\mathcal{c}^{-}}$-spacing measurements for (b1). (c2,c3) The $d_{M^{-}}$and $d_{\mathcal{c}^{-}}$-spacing measurements for $(\mathrm{c} 1)$. (d2,d3) The $d_{M^{-}}$and

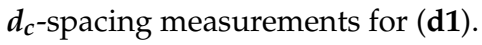

We obtained the average $d_{M}=0.2778 \mathrm{~nm}$ and $d_{c}=0.5240 \mathrm{~nm}$ for HR07, giving to $\theta=27.93^{\circ}$. For HR08, average $d_{M}=0.2772 \mathrm{~nm}$ and $d_{c}=0.5244 \mathrm{~nm}$, and $\theta=27.86^{\circ}$. For HR09, average $d_{M}=0.2771 \mathrm{~nm}$ and $d_{c}=0.5241 \mathrm{~nm}$, and $\theta=27.87^{\circ}$. For HR10, average $d_{M}=0.2766 \mathrm{~nm}$ and $d_{c}=0.5242 \mathrm{~nm}$, while $\theta=27.82^{\circ}$. The lateral lattice relaxation of the $2 \mathrm{D}$ aggregation looks slighter than the vertical relaxation of 3D stacked growth along the $c$-axis. We converted the lattice constants in $\AA$ units (i.e., $\boldsymbol{d}_{M}=\sqrt{3} a / 2$ and $\boldsymbol{d}_{c}=c$ ) of sample A (growth temperature $=620^{\circ} \mathrm{C}$ ) from the measured $d$-spacing with standard deviation in Table 1 . The vertical lattice relaxation as a function of stacked height $(z)$ can be easily observed from the plot of constants $a$ and $c$ against stacked height $(z)$ along the $c$-axis from HR01 to HR06, as shown by the black squares in Figure 4.

We also analyzed samples B $\left(630^{\circ}\right)$ and $C\left(640^{\circ}\right)$ in the same way to evaluate the effect of growth temperature on the stacked lattice relaxation of GaN microdisk. Some of the high-resolution TEM images and $\boldsymbol{d}$-spacing measurements are shown in Figures 5 and 6. Table 2 presents data for the 
obtained average $d_{M}$ and $d_{c}$ values, as well as those for $\theta$. Again, we converted the lattice constants $\left(\boldsymbol{a}\right.$ and $\boldsymbol{c}$ ) of sample B $\left(630^{\circ}\right)$ from the measured $\boldsymbol{d}$-spacing, as well as for sample C $\left(640^{\circ}\right)$. TEM Images corresponding to locations in the range HR11 to HR17 are also presented. Figure 5a1 shows location HR11 from Figure 1f. Figure 5b1 is HR13, Figure 5c1 is HR15, and Figure 5d1 is HR17.

Table 1. The lattice constants of sample A.

\begin{tabular}{ccccc}
\hline Sample A & Lattice Constant $\boldsymbol{a}(\AA)$ & $\begin{array}{c}\text { Standard } \\
\text { Deviation } \\
\text { of } \boldsymbol{a}(\AA)\end{array}$ & Lattice Constant $\boldsymbol{c}(\AA)$ & $\begin{array}{c}\text { Standard } \\
\text { Deviation } \\
\text { of } \boldsymbol{c}(\AA)\end{array}$ \\
\hline HR01 & 3.168 & 0.005 & 5.241 & 0.015 \\
HR02 & 3.174 & 0.004 & 5.237 & 0.014 \\
HR03 & 3.185 & 0.006 & 5.242 & 0.014 \\
HR04 & 3.191 & 0.006 & 5.241 & 0.008 \\
HR05 & 3.196 & 0.007 & 5.238 & 0.013 \\
HR06 & 3.200 & 0.005 & 5.241 & 0.002 \\
HR07 & 3.208 & 0.005 & 5.240 & 0.018 \\
HR08 & 3.201 & 0.009 & 5.244 & 0.010 \\
HR09 & 3.200 & 0.009 & 5.242 & 0.012 \\
HR10 & 3.194 & 0.013 & 5.241 & 0.011 \\
\hline
\end{tabular}

Table 2. The lattice constants of sample B.

\begin{tabular}{ccccc}
\hline Sample B & Lattice Constant $\boldsymbol{a}(\AA)$ & $\begin{array}{c}\text { Standard } \\
\text { Deviation } \\
\text { of } \boldsymbol{a}(\AA)\end{array}$ & Lattice Constant $\boldsymbol{c}(\AA)$ & $\begin{array}{c}\text { Standard } \\
\text { Deviation } \\
\text { of } \boldsymbol{c}(\AA)\end{array}$ \\
\hline HR11 & 3.165 & 0.005 & 5.246 & 0.003 \\
HR12 & 3.172 & 0.005 & 5.243 & 0.008 \\
HR13 & 3.184 & 0.004 & 5.237 & 0.003 \\
HR14 & 3.191 & 0.004 & 5.242 & 0.001 \\
HR15 & 3.201 & 0.003 & 5.245 & 0.004 \\
HR16 & 3.204 & 0.004 & 5.248 & 0.007 \\
HR17 & 3.206 & 0.004 & 5.244 & 0.007 \\
\hline
\end{tabular}

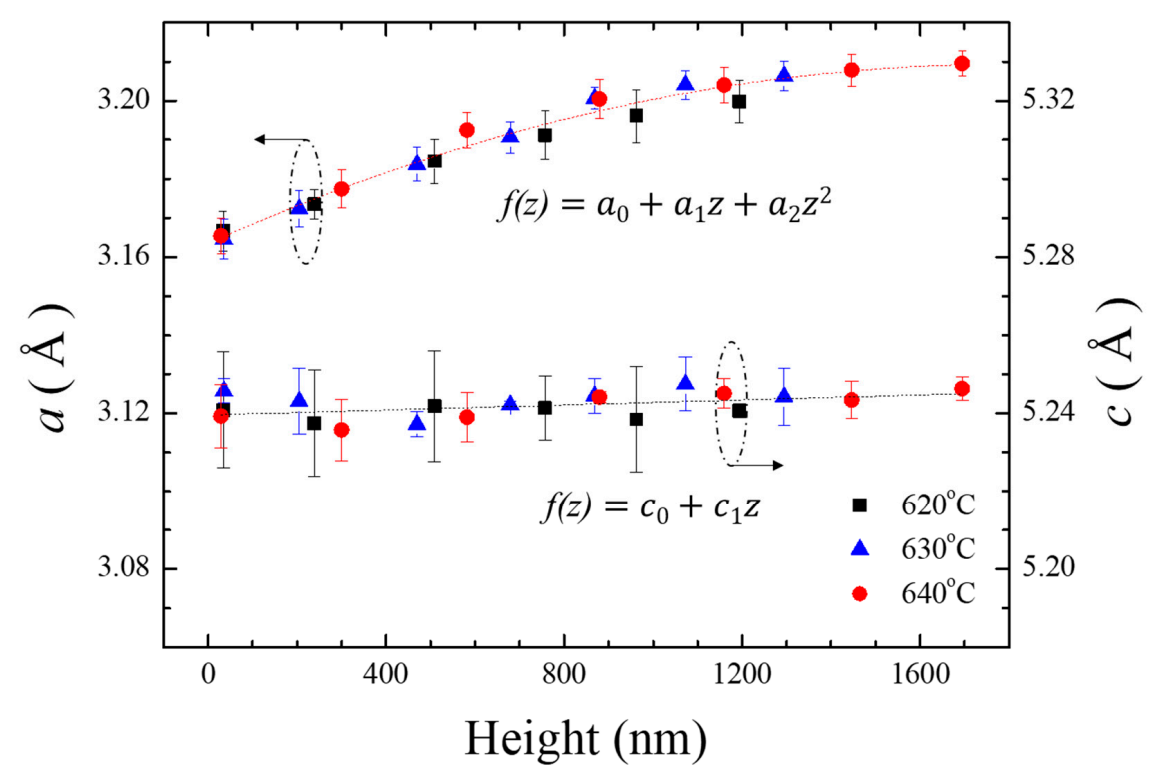

Figure 4. (Color online) The plots of lattice constants $\boldsymbol{a}$ and $c$ from Table 1, Table 2, and Table 3 versus stacked height $(z)$ along $c$-axis for samples A, B, and C. 
Table 3. The lattice constants of sample C.

\begin{tabular}{ccccc}
\hline Sample C & Lattice Constant $\boldsymbol{a}(\AA)$ & $\begin{array}{c}\text { Standard } \\
\text { Deviation } \\
\text { of } \boldsymbol{a}(\AA)\end{array}$ & Lattice Constant $\boldsymbol{c}(\AA)$ & $\begin{array}{c}\text { Standard } \\
\text { Deviation } \\
\text { of } \boldsymbol{c}(\AA)\end{array}$ \\
\hline HR18 & 3.165 & 0.005 & 5.239 & 0.008 \\
HR19 & 3.178 & 0.005 & 5.236 & 0.008 \\
HR20 & 3.193 & 0.005 & 5.239 & 0.006 \\
HR21 & 3.200 & 0.005 & 5.244 & 0.001 \\
HR22 & 3.204 & 0.004 & 5.245 & 0.004 \\
HR23 & 3.208 & 0.004 & 5.243 & 0.005 \\
HR24 & 3.210 & 0.003 & 5.246 & 0.003 \\
\hline
\end{tabular}
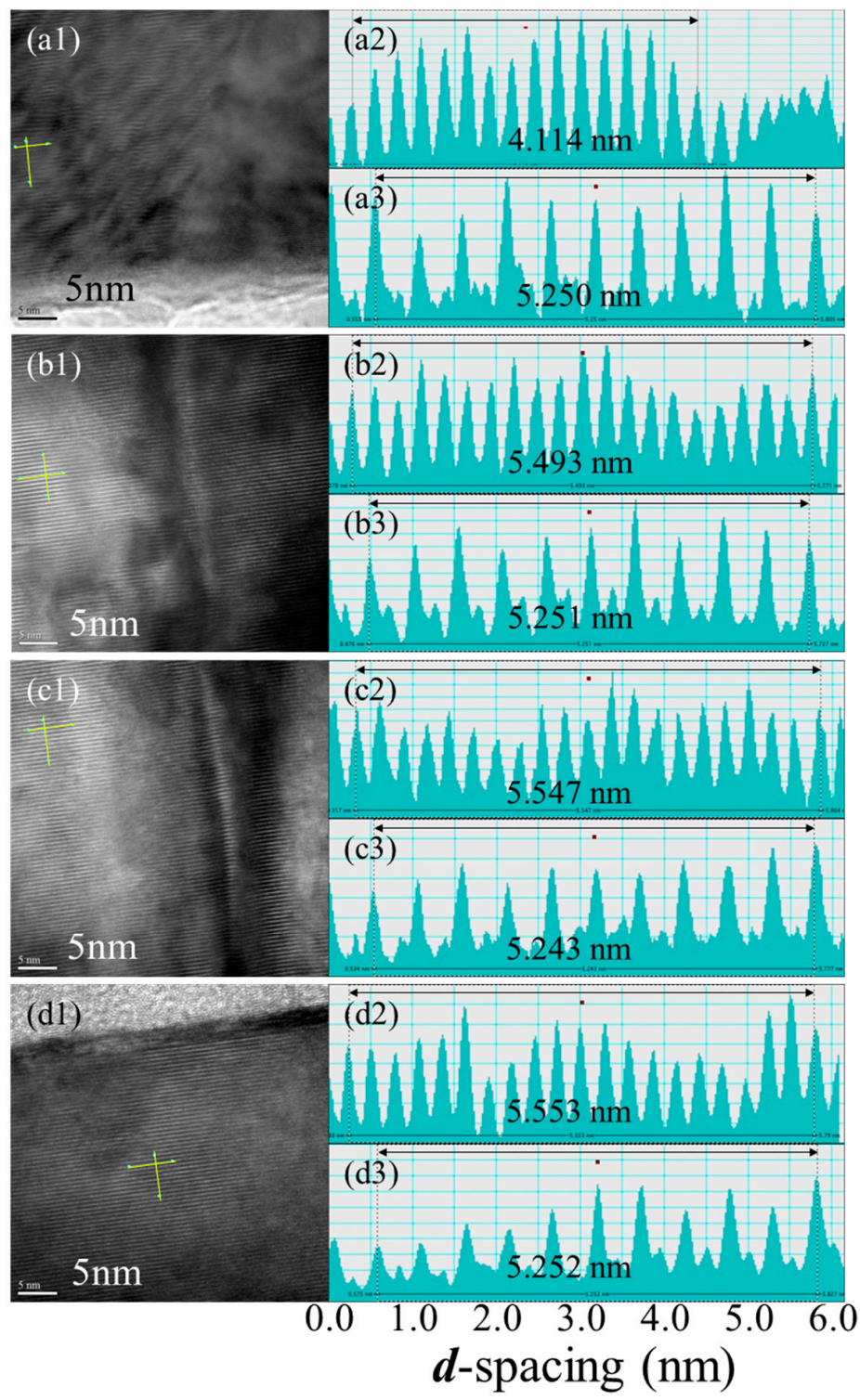

Figure 5. (a1,b1,c1,d1) High-resolution TEM images at HR11, HR13, HR15, and HR17, with scale bar of $5 \mathrm{~nm}$. $(\mathbf{a} 2, \mathrm{a} 3)$ The $d_{M^{-}}$and $d_{\mathcal{c}^{-}}$-spacing measurements for $(\mathbf{a} 1) .(\mathbf{b} 2, \mathrm{~b} 3)$ The $d_{M^{-}}$and $d_{c^{-}}$-spacing measurements for (b1). (c2,c3) The $d_{M^{-}}$and $d_{\mathcal{c}^{-}}$-spacing measurements for $(\mathbf{c} 1)$. (d2,d3) The $\boldsymbol{d}_{M^{-}}$and $d_{c^{-}}$spacing measurements for $(\mathbf{d} 1)$. 

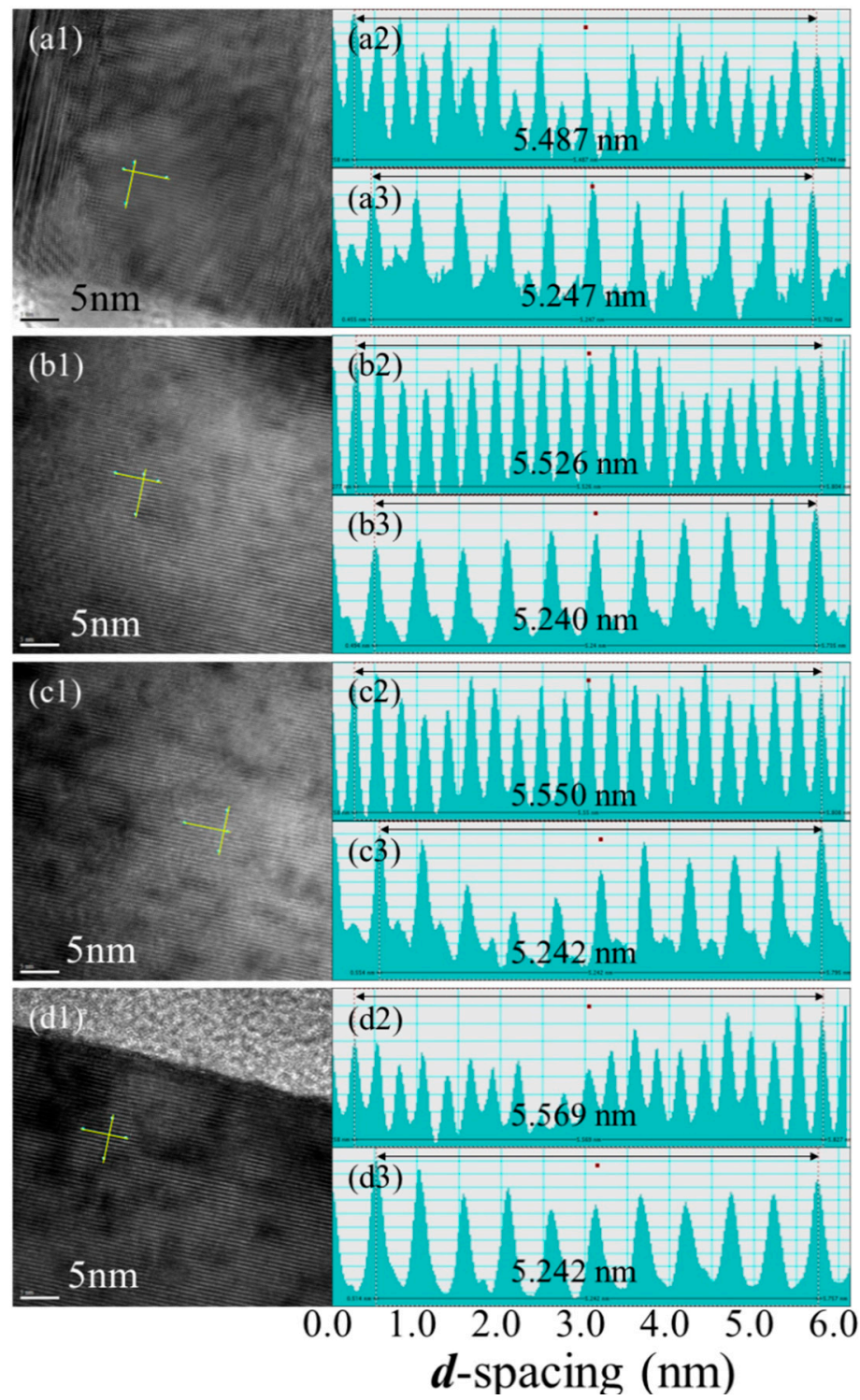

Figure 6. (a1,b1,c1,d1) High-resolution TEM images at HR18, HR20, HR22, and HR24, with scale bar of $5 \mathrm{~nm}$. (a2,a3) The $\boldsymbol{d}_{M^{-}}$and $\boldsymbol{d}_{c^{-}}$-spacing measurements for (a1). (b2,b3) The $\boldsymbol{d}_{M^{-}}$and $\boldsymbol{d}_{c^{-}}$-spacing measurements for (b1). (c2,c3) The $d_{M^{-}}$and $d_{c^{-}}$spacing measurements for (c1). (d2,d3) The $d_{M^{-}}$and $d_{c}$-spacing measurements for $(\mathbf{d} 1)$.

Values shown in Tables 1-3 were used to create fitting lines in Figure 4 by plotting constants $a$ and $c$ against stacked height $(z)$ along $c$-axis, as shown by blue triangles. Similarly, for sample C, the obtained $\boldsymbol{d}_{\boldsymbol{M}}, \boldsymbol{d}_{\boldsymbol{c}}$, and $\theta$ values are presented in Table 3 , with corresponding TEM images in Figure 6a1 (HR18), Figure 6b1 (HR20), Figure 6c1 (HR22), and Figure 6d1 (HR24). Lattice constants $\left(\boldsymbol{a}\right.$ and $\boldsymbol{c}$ ) for Sample $\mathrm{C}\left(640^{\circ}\right)$ are shown by red circles in Figure 4. From the best curve fitting results, we obtained a linear relationship for lattice constant $c$ : $f(z)=c_{0}+c_{1} z$, with $c_{0}=(5.240 \pm 0.001) \AA$ and $c_{1}=(3.213 \pm 1.376) \times 10^{-6}$; and a quadratic variation for lattice constant $a: f(z)=a_{0}+a_{1} z+a_{2} z^{2}$, with $a_{0}=(3.163 \pm 0.001) \AA, a_{1}=(5.114 \pm 0.356) \times 10^{-5}$ and $a_{2}=(-1.426 \pm 0.222) \times 10^{-8}$.

The datapoints for all three samples follow the fitting equations within the standard deviations marked by error bars. The strain $(\varepsilon)$ against stacked height $(z)$ can be defined as $\varepsilon=[f(z)-f(0)] / f(0)$ during the epi-growth. This indicates that the awl-shaped hexagonal GaN microdisks were grown under an anisotropic strain and the effect of temperature (from 620 to $640^{\circ} \mathrm{C}$ ) results in minor fluctuations in strain during the epi-growth. From the extrapolation of fitting curves, we obtained the initial lattice 
constants of the GaN microdisk at nucleation interface on the LAO substrate $(z=0 \mathrm{~nm}): a_{0}=3.163 \AA$ and $c_{0}=5.240 \AA$. Because the substrate $\left(\gamma-\mathrm{LiAlO}_{2}\right)$ is a tetragonal structure with lattice constants $a_{\mathrm{LAO}}=b_{\mathrm{LAO}}=5.169 \AA$ and $c_{\mathrm{LAO}}=6.268 \AA$, it provides a nearly lattice-matched rectangular anionic basal plane for $2 \mathrm{D} M$-plane $\mathrm{GaN}$ (1100) epi-growth, where $a_{\mathrm{LAO}} \cong c_{\mathrm{GaN}}$ (within $1.4 \%$ ) and $c_{\mathrm{LAO}} \cong$ $2 a_{\mathrm{GaN}}$ (within $0.9 \%$ ), as shown in the inset of Figure 7 [13]. In addition, the substrate also provides a hexagonal anionic basal plane (i.e., oxygen sites in the inset of Figure 7) for 3D c-plane GaN (000 $\overline{1})$ microdisk gowth [14].

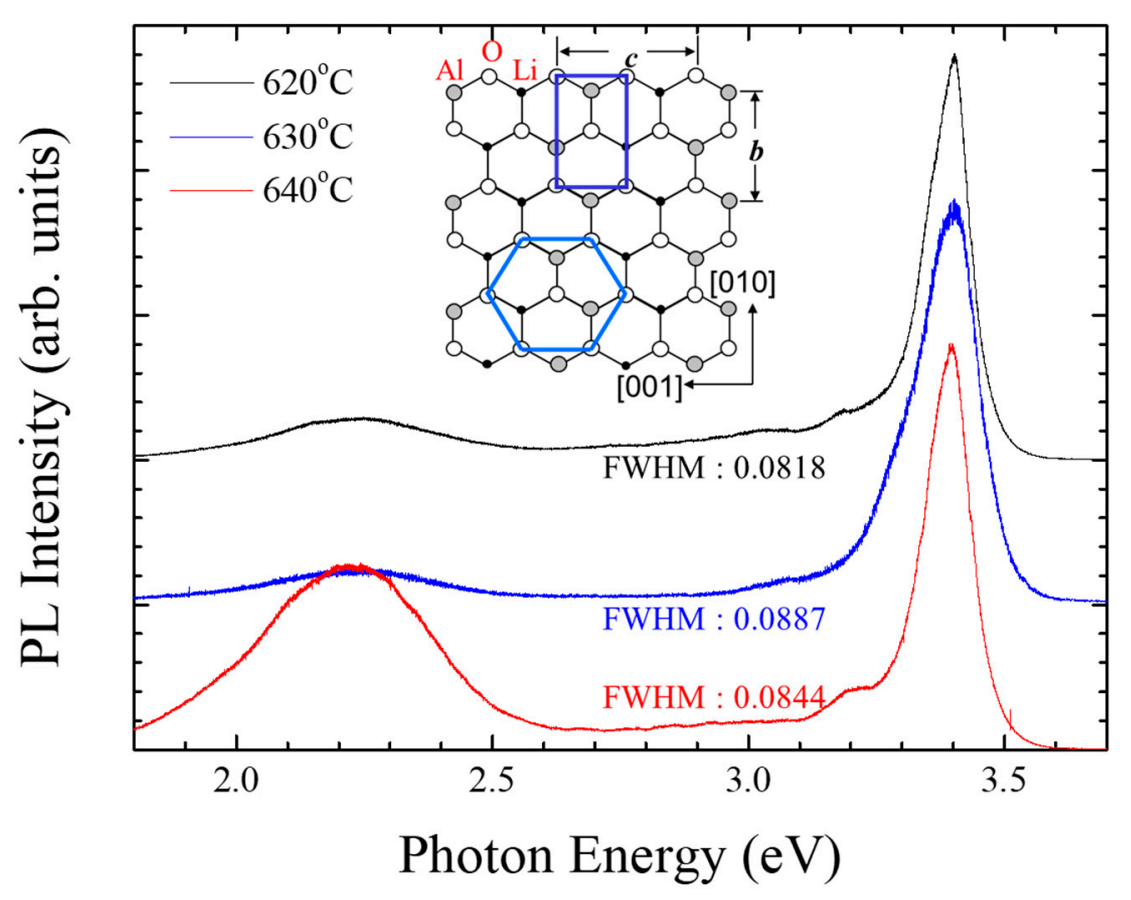

Figure 7. PL spectra at room temperature for samples A, B, and C. The inset shows the diagram of lattice structure for (100) $\gamma$-LAO substrate.

By controlling growth parameters, the 3D c-plane GaN (0001) microdisks can be easily achieved in our PAMBE system [9-11]. Therefore, the edge of the hexagonal basal plane is equal to $(1 / 2) c_{\mathrm{LAO}}=3.134 \AA$, which offers the hexagonal basis for the nucleation of the GaN microdisk $\left(a_{0}=3.163 \AA\right)$ with a $0.94 \%$ mismatch. This mismatch was the origin of the stress for further GaN microdisk growth, resulting in the strain with a linear relationship to stacked height $(z)$ in lattice constant $c$ and $a$ quadratic deviation in lattice constant $a$. We also checked the optical property of the samples by photoluminescence (PL) measurements at room temperature, as shown in Figure 7. We took PL measurements with a He-Cd $325 \mathrm{~nm}$ laser as a light source and focused the laser beam on center of microdisk. The detailed analyses can be seen in our previous study in reference [10]. The major peaks at $3.4 \mathrm{eV}$ referred to the band edge transition of $\mathrm{GaN}$, and exhibited no difference among samples, and only a slight change occurs in their full width at half maximum (FWHM). The other peaks (e.g., 2.23 and $3.22 \mathrm{eV}$ ) were attributed to the emission of excitons bound to the structured defects of microstructures [15-17] near the microdisks, as shown in Figure 1b,e,h.

\section{Conclusions}

We studied the lattice relaxation of self-assembled GaN microdisks grown by PAMBE on a $\gamma-\mathrm{LiAlO}_{2}$ substrate. We obtained the anisotropic strain of the GaN microdisk against stacked height (z); a linear relationship for vertical strain of lattice constant $c: f(z)=c_{0}+c_{1} z$, with $c_{0}=5.240 \AA$ and $c_{1}=3.213 \times 10^{-6}$; and a quadratic variation for lateral strain of lattice constant $a: f(z)=a_{0}+a_{1} z+a_{2} z_{2}$, with $a_{0}=3.163 \AA, a_{1}=5.114 \times 10^{-5}$ and $a_{2}=-1.427 \times 10^{-8}$. The lattice mismatch is about $0.94 \%$ at the 
interface between GaN microdisks and the $\gamma-\mathrm{LiAlO}_{2}$ substrate, which induces the anisotropic strain during the epi-growth. The 3D self-assembling $28^{\circ}$ growth of $\mathrm{GaN}$ microdisk created the unbalanced stress between $\{000 \overline{1}\}$ planes, leading to the anisotropic strain during the layer-by-layer self-assembly. The change in growth temperature (from 620 to $640^{\circ} \mathrm{C}$ ) only slightly influences the lattice relaxation at the hetero-interface between $\mathrm{GaN}$ and $\mathrm{LAO}$ substrates, as well as the microstructure of $\mathrm{GaN}$ microdisks. The strain relationship fitting provides important information for the theoretical band calculation, as well as for engineering $\operatorname{In}_{x} \mathrm{Ga}_{1-\mathrm{x}} \mathrm{N} / \mathrm{GaN} \mathrm{QW}$ micro-LEDs.

Author Contributions: Conceptualization was done by I.L. and H.-Y.Y.; the experiments were performed and analyzed by H.-Y.Y., C.-D.T., H.-J.S. and H.-C.H.; the LAO substrate was provisioned by M.M.C.C.; technical assistance provided by Y.-C.W., L.H., T.W. and C.T.C.K.; the original draft preparation was done by H.-Y.Y.; the review and editing was done by I.L. All authors have read and agreed to the published version of the manuscript.

Funding: This research was funded by the Ministry of Science and Technology (MOST), grant number 108-2811-M-110-528.

Acknowledgments: The authors would like to thank C.-C. Yang, Y.-C. Lin, C.-H. Hu and S.-T. You for their assistance. The project was supported by the Ministry of Science and Technology of Taiwan (MOST, Taiwan) and the Core Facilities Laboratory for Nanoscience and Nanotechnology in Kaohsiung and Pingtung Area.

Conflicts of Interest: The authors declare no conflict of interest.

\section{References}

1. Lo, I.; Tsai, J.K.; Yao, W.J.; Ho, P.C.; Tu, L.-W.; Chang, T.C.; Elhamri, S.; Mitchel, W.C.; Hsieh, K.Y.; Huang, J.H.; et al. Spin splitting in modulation-doped $\mathrm{Al}_{\mathrm{x}} \mathrm{Ga}_{1-\mathrm{x}} \mathrm{N} / \mathrm{GaNheterostructures.} \mathrm{Phys.} \mathrm{Rev.} \mathrm{B} \mathrm{2002,}$ 65, 161306. [CrossRef]

2. Lo, I.; Wang, W.T.; Gau, M.H.; Tsay, S.F.; Chiang, J.C. Wurtzite structure effects on spin splitting inGaN/AlNquantum wells. Phys. Rev. B 2005, 72, 245329. [CrossRef]

3. Nakamura, S.; Senoh, M.; Iwasa, N.; Nagahama, S.-I.; Yamada, T.; Mukai, T. Superbright Green InGaN Single-Quantum-Well-Structure Light-Emitting Diodes. Jpn. J. Appl. Phys. 1995, 34, L1332. [CrossRef]

4. Nakamura, S.; Pearton, S.; Fasol, G. The Blue Laser Diode, 2nd ed.; Springer: Berlin, Germany, 2000.

5. Madelung, O. Data in Science and Technology, Semiconductors Group IV Elements and III-V Compounds; Springer: Berlin, Germany, 1991.

6. Morkoc, H.; Strite, S.; Gao, G.B.; Lin, M.E.; Sverdlov, B.; Burns, M. Large-band-gap SiC, III-V nitride, and II-VI ZnSe-based semiconductor device technologies. J. Appl. Phys. 1994, 76, 1363. [CrossRef]

7. Wu, J.; Walukiewicz, W.; Yu, K.M.; Ager, J.W.; Haller, E.E.; Lu, H.; Schaff, W.J.; Saito, Y.; Nanishi, Y. Unusual properties of the fundamental band gap of InN. Appl. Phys. Lett. 2002, 80, 3967-3969. [CrossRef]

8. Wu, J.; Walukiewicz, W.; Shan, W.; Yu, K.M.; Ager, J.W.; Li, S.X.; Haller, E.E.; Lu, H.; Schaff, W.J. Temperature dependence of the fundamental band gap of InN. J. Appl. Phys. 2003, 94, 4457-4460. [CrossRef]

9. Lo, I.; Hsieh, C.-H.; Hsu, Y.-C.; Pang, W.-Y.; Chou, M.-C. Self-assembled GaN hexagonal micropyramid and microdisk. Appl. Phys. Lett. 2009, 94, 062105. [CrossRef]

10. Tsai, C.-D.; Lo, I.; Wang, Y.-C.; Yang, C.-C.; You, S.-T.; Yang, H.-Y.; Huang, H.-C.; Chou, M.M.C. Finite growth of InGaN/GaN triple-quantum-well microdisks on $\mathrm{LiAlO}_{2}$ substrate. AIP Adv. 2018, 8, 095208. [CrossRef]

11. Tsai, C.; Lo, I.; Wang, Y.; Yang, C.; Yang, H.; Shih, H.; Huang, H.; Chou, M.M.C.; Huang, L.; Tseng, B. Indium-Incorporation with $\mathrm{In}_{\mathrm{x}} \mathrm{Ga}_{1-\mathrm{x}} \mathrm{N}$ Layers on $\mathrm{GaN}$-Microdisks by Plasma-Assisted Molecular Beam Epitaxy. Crystals 2019, 9, 308. [CrossRef]

12. Lo, I.; Wang, Y.-C.; Hsu, Y.-C.; Shih, C.-H.; Pang, W.-Y.; You, S.-T.; Hu, C.-H.; Chou, M.M.C.; Hsu, G.Z.L. Electrical contact for wurtzite GaN microdisks. Appl. Phys. Lett. 2014, 105, 082101.

13. Waltereit, P.; Brandt, O.; Trampert, A.; Grahn, H.T.; Menniger, J.; Ramsteiner, M.; Reiche, M.; Ploog, K.H. Nitride semiconductors free of electrostatic fields for efficient white light-emitting diodes. Nat. Cell Biol. 2000, 406, 865-868. [CrossRef] [PubMed]

14. Hsieh, C.H.; Lo, I.; Gau, M.H.; Chen, Y.L.; Chou, M.C.; Pang, W.Y.; Chang, Y.I.; Hsu, Y.C.; Sham, M.W.; Chiang, J.C.; et al. Self-assembled c-plane GaN nanopillars on $\gamma-\mathrm{LiAlO}_{2}$ substrate grown by plasma-assisted molecular-beam epitaxy. Jpn. J. App. Phys. 2008, 47, 891. [CrossRef]

15. Reshchikov, M.A.; Morkoç, H. Luminescence properties of defects in GaN. J. Appl. Phys. 2005, 97, 061301. [CrossRef] 
16. Reshchikov, M.A.; Huang, D.; Yun, F.; He, L.; Morkoç, H.; Reynolds, D.C.; Park, S.S.; Lee, K.Y. Photoluminescence of GaN grown by molecular-beam epitaxy on a freestanding GaN template. Appl. Phys. Lett. 2001, 79, 3779-3781. [CrossRef]

17. Díaz-Guerra, C.; Piqueras, J.; Volciuc, O.; Popa, V.; Tiginyanu, I.M. Cathodoluminescence microscopy and spectroscopy of GaN epilayers microstructured using surface charge lithography. J. Appl. Phys. 2006, 100, 023509. [CrossRef]

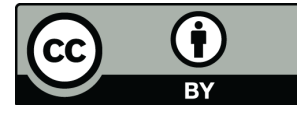

(C) 2020 by the authors. Licensee MDPI, Basel, Switzerland. This article is an open access article distributed under the terms and conditions of the Creative Commons Attribution (CC BY) license (http://creativecommons.org/licenses/by/4.0/). 